\title{
Factors that may influence polymorphous low-grade adenocarcinoma growth
}

\author{
Andresa Borges Soares ${ }^{1} \cdot$ Elizabeth Ferreira Martinez ${ }^{1}$. \\ Patricia Fernandes Avila Ribeiro ${ }^{1}$ - Icleia Siqueira Barreto ${ }^{1}$. Maria Cássia Aguiar ${ }^{2}$. \\ Cristiane Furuse $^{3} \cdot$ Marcelo Sperandio $^{1}$ • Victor Angelo Montalli ${ }^{1}$. \\ Ney Soares de Araújo ${ }^{1}$ - Vera Cavalcanti de Araújo ${ }^{1}$
}

Received: 23 November 2016/Revised: 20 December 2016 / Accepted: 30 January 2017 /Published online: 10 February 2017

(C) Springer-Verlag Berlin Heidelberg 2017

\begin{abstract}
There is mounting evidence on the importance of some biological processes in tumor growth, such as vascular supply, apoptosis, autophagy, and senescence. We have investigated these processes in polymorphous low-grade adenocarcinoma (PLGA), in an attempt to identify those that are relevant for this particular lesion. We analyzed 31 cases of PLGA using immunohistochemistry to antibodies against CD34 and CD105 to detect blood vessels; against D2-40 to detect lymphatic vessels; against $\mathrm{Bax}, \mathrm{Bcl}-2$, and survivin to explore cell apoptosis; and against Beclin and LCB3 to investigate autophagy and against p21 and p16 to assess senescence. Our results showed that PLGA growth does not depend on newly formed vessels but only on preexisting vasculature. Furthermore, PLGA is promoted by autophagy, sustained by both anti-apoptotic and anti-senescence signals, and stimulated by Bcl-2 and survivin.
\end{abstract}

Keywords Polymorphous low-grade adenocarcinoma . Angiogenesis · Apoptosis · Autophagy $\cdot$ Senescence $\cdot$ Bcl2 . Survivin $\cdot$ LC3B

Andresa Borges Soares

andresabs@hotmail.com

1 Department of Oral Pathology, São Leopoldo Mandic Institute and Research Center, Rua José Rocha Junqueira, 13 Ponte Preta, Campinas, SP 13045-755, Brazil

2 Department of Oral Pathology, Federal University of Minas Gerais, Belo Horizonte, MG, Brazil

3 Department of Oral Pathology, State University of São Paulo, Araçatuba, SP, Brazil

\section{Background}

Polymorphous low-grade adenocarcinoma (PLGA) is a malignant neoplasm almost exclusively of the minor salivary glands, characterized by cytologic uniformity, morphologic diversity, an infiltrative growth pattern, and low metastatic potential [1]. PLGA was first identified as a specific salivary gland adenocarcinoma almost simultaneously in 1983 by Freedman and Lumerman [2] and Batsakis et al. 1983 [3]. Investigations have since focused mostly on the clinical behavior of the disease which, despite a low risk of metastasis, presents a high risk of morbidity in addition to the potentially disfiguring nature of the treatment [4].

In order to elucidate how PLGA lesions grow, we assessed some factors deemed essential for tumor growth, such as vascularization, apoptosis, autophagy, and senescence.

Angiogenesis and lymphangiogenesis within the tumor microenvironment are processes that can influence neoplastic growth and metastatic dissemination. Investigating vascularization, imbalances between the mechanisms of cell regulation, proliferation, and death may shed some light on key features of PLGA and contribute to the current knowledge on its growth $[5,6]$.

Programmed cell death, which may occur through apoptosis, autophagy, or programmed necrosis, plays an important role in the maintenance of tissue homeostasis, including removal of damaged cells by controlling cell proliferation and death under physiological conditions. In cancer, however, this balance is lost and deregulation of programmed cell death is associated with various stages of carcinogenesis [7]. It has been recognized that apoptosis plays multiple roles in cancer development, growth, and response to treatment [8]. Loss of cell proliferation control and resistance to apoptosis are important mechanisms in tumor growth. Deregulated expression of proteins that control apoptosis can suppress elimination of 
DNA-damaged cells, which in turn will contribute to accumulation of oncogenic mutations, thus facilitating malignant transformation and tumor development [9].

Autophagy is a critical cellular mechanism that can promote cell survival under many circumstances. Overactivation of autophagy pathways can, however, lead to cell death $[7,10,11]$. Several studies have demonstrated multidirectional communication between autophagy and carcinogenesis $[12,13]$. Autophagy can slow or accelerate the development of tumors, depending on their type, microenvironment and stage [14].

It has recently been reported that autophagy has a dual role in carcinogenesis: (1) a supervising mechanism protecting normal cells from malignant transformation by recycling damaged organelles, aggregated proteins, mitochondrial abnormalities, damaged DNA, and (2) a mechanism supporting tumor growth by providing nutrients that are critical to tumor cell metabolism [13].

Senescence is a terminal proliferation arrest mechanism usually related to the aging process. Cumulative evidence, however, has shown that cellular senesence exerts a significant impact on tumourigenesis and degenerative disease [15-17]. Along with apoptosis, it is considered a failsafe mechanism that prevents cells from transforming into a malignant phenotype.

The aim of this study was to investigate the expression of a panel of biomarkers in PLGA by immunohistochemistry, based on the above-described processes that regulate cell death. Ultimately, the results might provide insight into the possible mechanisms involved in the growth of this particular entity.

\section{Materials and methods}

\section{Immunohistochemistry}

This study was approved by the Research Ethics Committee of the Sao Leopoldo Mandic Dental School and Research Institute, protocol number 42315715.1.0000.5374

Thirty-one cases of PLGA, previously characterized both morphologically and by immunohistochemistry, composed our sample population. Cases were included based on diagnosis and on a negative history of previous oncological treatment. In our series, no cases of cribriform adenocarcinoma of minor salivary glands (CAMSG) were included, as no cases in our series corresponded to the criteria for a diagnosis of CAMSG.

Immunohistochemical staining was performed using the following antibodies (Table 1): anti-CD34 and anti-CD105 to detect blood vessels; anti-D2-40 to detect lymphatic vessels; anti-Bax, anti-Bcl-2, and anti-survivin to detect cell apoptosis; anti-Beclin and anti-LCB3 to detect autophagy; and anti-p21 and anti-p16 to detect senescence.

From formalin-fixed paraffin-embedded tumor tissue samples, $5 \mu \mathrm{m}$ sections were cut and mounted, dewaxed, and rehydrated and endogenous peroxidase activity was quenched
Table 1 Details of the antibodies used for immunohistochemistry

\begin{tabular}{cclll}
\hline Specificity & Clone & Dilution & Source & Buffer (AR) \\
\hline CD34 & QBEnd 10 & $1: 50$ & Dako $^{\mathrm{a}}$ & Citrate \\
CD 105 & SNG & $1: 10$ & Dako $^{\mathrm{a}}$ & Pepsin \\
D2-40 & D2-40 & $1: 200$ & Dako $^{\mathrm{a}}$ & Tris-EDTA \\
VEGF & Sc-7269 & $1: 100$ & Santa Cruz $^{\mathrm{b}}$ & Citrate \\
BAX & 6A7 & $1: 150$ & Abcam $^{\mathrm{c}}$ & Citrate \\
Bc12 & 124 & $1: 150$ & Dako $^{\mathrm{a}}$ & Citrate \\
Survivin & Ab24479 & $1: 50$ & Abcam $^{\mathrm{c}}$ & Citrate \\
Beclin & EPR1733Y & $1: 50$ & Abcam $^{\mathrm{c}}$ & Citrate \\
LC3B & Ab51520 & $1: 100$ & Abcam $^{\mathrm{c}}$ & Citrate \\
p21 & Ab18209 & $1: 100$ & Abcam $^{\mathrm{c}}$ & Citrate \\
p16 & G175-405 & $1: 50$ & Zeta Corporation $^{\mathrm{d}}$ & Citrate \\
\hline
\end{tabular}

${ }^{a}$ Dako Corporation, Glostrup, Denmark

${ }^{\mathrm{b}}$ Santa Cruz Biotechnogy, Inc., Santa Cruz, CA, USA

${ }^{\mathrm{c}}$ Abcam, Cambridge, UK

${ }^{\mathrm{d}}$ Zeta Corporation, Sierra Matre, CA, USA

by immersing slides in 3\% hydrogen peroxide. Antigen retrieval (AR) was achieved by immersing the slides in boiling citrate buffer ( $\mathrm{pH}$ 6.0) for staining for CD34, Bax, BCl-2, survivin, Beclin, LC3B, p21, and p16. For CD105 staining, AR was performed using $0.4 \%$ pepsin, while for D2-40, TrisEDTA was used (Table 1). Only the sections for CD105 staining were incubated at $37{ }^{\circ} \mathrm{C}$ with a serum-free protein blocking solution (code x0909, Dako, SA, Denmark) for $30 \mathrm{~min}$. Subsequently, the sections were incubated with the primary antibody for $60 \mathrm{~min}$ (anti-CD34, anti-Bax, anti-Bcl-2, anti-survivin, anti-Beclin, anti-LC3B, anti-P21) or overnight (anti-Cd105, anti-D2-40, anti-P16) at $4{ }^{\circ} \mathrm{C}$ followed by EnVision polymer HRP and Envision+ (code K1491, DAKO, SA, Denmark) for $1 \mathrm{~h}$ at $37^{\circ} \mathrm{C}$. The sections were developed for $5 \mathrm{~min}$ at $37^{\circ} \mathrm{C}$ with $3.3^{\prime}$ - diaminobenzidine tetrahydrochloride (DAB) and counter-stained with hematoxylin. A negative control was obtained by omitting the primary antibody. No staining was observed on this section.

As incision biopsies only provide a limited amount of tissue for analysis, some paraffin blocks did not contain sufficient material for all immunohistochemistry reactions, hence the different numbers reported in the results.

\section{Density of blood and lymphatic microvessels}

For all 31 cases, immunohistochemical staining for CD34, CD105, and D2-40 was interpreted by two experienced pathologists (VCA and ABS) using a double-headed microscope. Vascularization was assessed on all specimen, though only the highest cellular areas were selected for blood vessel quantification. In order to achieve this, images were obtained from 5 fields (hotspots) per case ( $\times 40$ objective, $0.44 \mathrm{~mm}$ field 
diameter) using a digital camera (Infinity 1, Canada) attached to an Olympus CX30 microscope. The images were evaluated using the Imagelab analysis software (version 2.4), which allowed manual segmentation of the target vessels. Blood and lymphatic microvascular densities obtained from CD34, CD105, and D2-40 immunostaining were expressed as the mean number of intratumoural microvessels counted. There was no restriction regarding the size of a countable microvessel, although vessels presenting muscle walls were not counted and necrotic areas were excluded.

\section{Cell apoptosis, autophagy and senescence}

Immunohistochemical staining for Bax yielded an assessable result in 17 cases, for Bcl-2 in 29 cases, and for survivin in 29 cases. A ratio of Bax and Bcl2 staining was calculated on the 17 cases in which both Bax and Bcl 2 were expressed. Expression of the autophagy-associated marker Beclin and LC3B was evaluated in 23 and 20 cases, respectively. Expression of cell senescence-associated markers p21 and p16 was analyzed in 23 and 30 cases, respectively. Immunostained sections were evaluated qualitatively and semi-quantitatively. Qualitative analysis was performed based on the proportion of positive neoplastic cells relative to all neoplastic cells throughout the tissue section, with $10 \%$ as cut-off between negative $(<10 \%)$ and positive cases. Semi-quantitative analysis was performed by assigning cases with less than $10 \%$ positive cells score 0,10 $25 \%$ positive cells score $1,25-50 \%$ score 2 , and higher than $50 \%$ positive cells score 3 .

\section{Results}

The 31 patients included in this study had a mean age of 57.8 years and were mostly female $(71 \%)$. Data regarding patient demographics and site of the lesions are listed in Table 2.

\section{Assessed of microvascular density and lymphatic vessel density using anti-D34, CD105, and D2-40 staining}

High vascular density was observed in PLGA, with vessels distributed throughout the tumor, both in tumor cell masses and in the stroma. Immunohistochemical staining showed that these vessels were mostly CD34 positive (mean $=12.62$ ), whereas few if any were CD105 positive $($ mean $=0.34)($ Fig. 1a, $b)$. Only a small number of D2-40-positive (lymphatic) vessels were observed $($ mean $=0.96)($ Fig. 1c) $($ Fig. 2a) .

\section{Expression of apoptosis markers Bax, Bcl2, and survivin}

Bax expression by immunohistochemistry was granular cytoplasmic but remarkably heterogeneous, varying from weak or negative to positive or intense. Six cases (35\%)
Table 2 Clinicopathological findings of PLGA

\begin{tabular}{llll}
\hline Case & Age (year) & Gender & Site \\
\hline 1 & 72 & M & Palate \\
2 & 50 & F & Palate \\
3 & 62 & F & Buccal mucosa \\
4 & 48 & F & Palate \\
5 & 51 & F & Palate \\
6 & 35 & F & Palate \\
7 & 83 & F & Palate \\
8 & 48 & F & Buccal mucosa \\
9 & 57 & M & Palate \\
10 & 68 & F & Upper buccal sulcus \\
11 & 66 & M & Buccal mucosa \\
12 & 73 & F & Upper lip \\
13 & 51 & F & Lower buccal sulcus \\
14 & 67 & F & Upper lip \\
15 & 70 & M & Palate \\
16 & 52 & F & Palate \\
17 & 69 & F & Palate \\
18 & 58 & M & Palate \\
19 & 77 & M & Alveolar ridge and palate \\
20 & 41 & M & Maxilla \\
21 & 36 & F & NA \\
22 & 65 & F & Palate \\
23 & 55 & F & Palate \\
24 & 70 & F & Palate \\
25 & 68 & F & Alveolar ridge \\
26 & 45 & F & Alveolar ridge \\
27 & 64 & Palate \\
28 & 41 & Palate \\
30 & 51 & 33 & Fper lip \\
\hline 31 & 67 & F & \\
\hline
\end{tabular}

$F$ female, $M$ male, $N A$ not available

scored 0 , three cases (18\%) scored 1, six cases (35\%) scored 2, and two cases (12\%) scored 3 (Fig. 1d). Strong membrane and cytoplasmic expression of $\mathrm{Bcl} 2$ was noted in tumor cells in all cases, with more than $50 \%$ of tumor cells positive (score 3) (Fig. 1e). Survivin expression was observed in both the nucleus and cytoplasm. Twenty-five cases (86\%) showed strong diffuse staining for survivin, with more than $50 \%$ of tumor cells staining (score 3 ). Score 2 was observed in two cases (7\%) and the remaining two cases (7\%) scored 1 (Fig. 1f) (Fig. 2b).

The calculated $\mathrm{Bax} / \mathrm{Bcl} 2$ ratio ranged from 0 to 1 , because all Bcl2-positive cases scored 3. The Bax-Bc12 ratio for most cases $(n=15)$ was less than 1 , suggesting that virtually no apoptosis was taking place. For the remaining two cases, the ratio was 1 . 
Fig. 1 PLGA

Immunohistochemistry panel.

Microvascular and lymphatic

vessels $(\mathbf{a}, \mathbf{b}, \mathbf{c})$ : a Expression of

CD34 showing numerous vessels

distributed throughout the tumor.

b CD105 positive in rare newly

formed vessels. $\mathrm{c}$ D2-40 positivity

in the peripheral area of the

tumor; note the absence of

lymphatic vessels in the bulk of

the tumor. Markers of cell

apoptosis (d, e, f): d Relatively

low immunoexpression of Bax, $\mathbf{e}$

intense $\mathrm{Bcl} 2$ expression, $\mathbf{f}$

survivin expression; note the

strong nuclear pattern of

immunoexpression. Autophagy

$(\mathbf{g}, \mathbf{h}): \mathbf{g}$ Beclin is expressed in

nearly all cells, $\mathbf{h}$ strong

cytoplasmic expression of LC3B.

Senescence $(\mathbf{i}, \mathbf{j})$ : i p 21 was rarely expressed in stromal cells and not at all in the parenchyma, $\mathbf{j}$ p16 is seldom observed in parenchymal cells
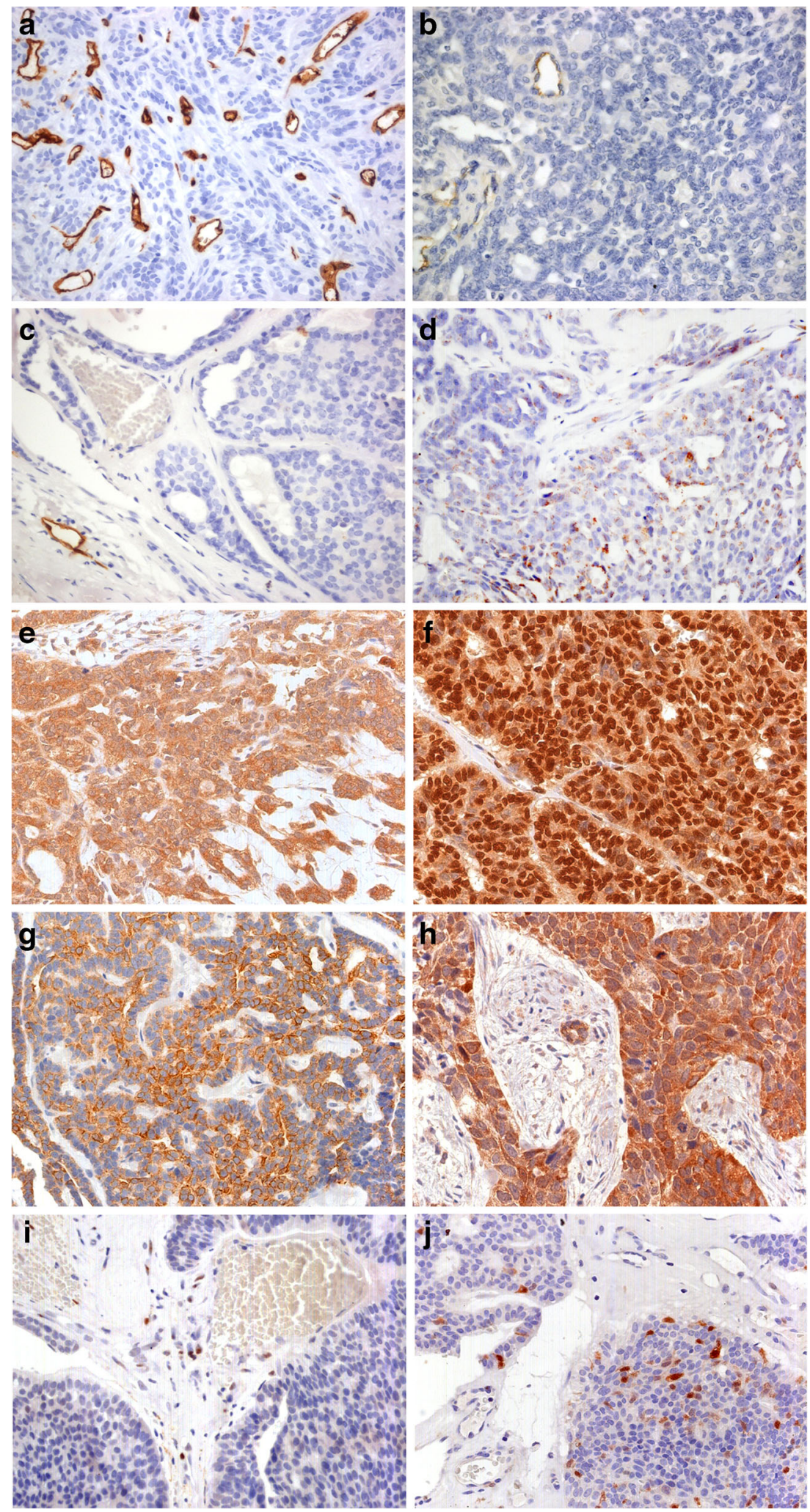

\section{Expression of autophagy markers Beclin and LC3B}

Membrane and cytoplasmic Beclin expression was remarkably heterogeneous, varying between weak or negative and positive or intense. Five cases (22\%) scored 0 , six cases $(26 \%)$ scored 1 , two cases $(9 \%)$ scored 2 , and 10 cases $(43 \%)$ scored 3 (Fig. 1g). Cytoplasmic LC3B expression was strong and diffuse in $17(85 \%)$ 

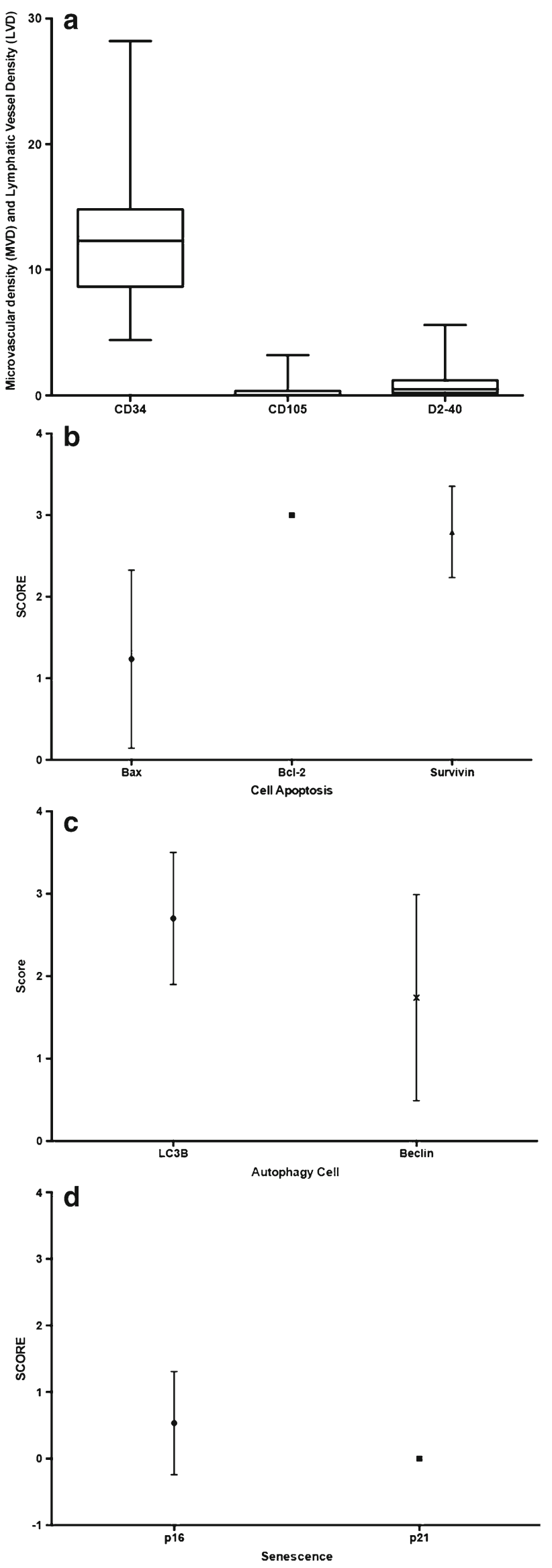

Fig. 2 Distribution of positive cell counts (mean \pm SD) or scores (median) associated to each set or immunohistochemical markers. a Box-plot of microvessel density (MVD cell counts) and lymphatic vessel density (LVD cell counts). B Median distribution of cell apoptosis based on Bax, Bcl2, and survivin. C Median distribution of the autophagy-related markers Beclin and LC3B. D Median distribution of the senescence-related markers p 21 and p16

cases (score 3), one case (5\%) each scored 0,1 , and 2 (Fig. 1h) (Fig. 2c).

\section{Expression of senescence markers p21 and p16}

In all cases, nuclear expression of $\mathrm{p} 21$ was entirely negative or in less than $10 \%$ of tumor cells (score 0 ) (Fig. 1i). Most cases were weakly positive for $\mathrm{p} 16$, all expression exclusively nuclear. Eighteen $(60 \%)$ cases scored 0, nine cases (30\%) scored 1 , two cases $(7 \%)$ scored 2 , and only one case $(3 \%)$ was strongly positive (score 3) (Fig. 1j) (Fig. 2d).

\section{Discussion}

There is controversy as to whether or not PLGA is a single pathological entity, as recent evidence shows that similar lesions with worse prognosis (notably cribriform adenocarcinoma of the tongue [18] and cribriform adenocarcinoma of the tongue and minor salivary glands (CAMSG) [19]) may present histologically with features overlapping those of PLGA. CAMSG is considered a unique entity, as it usually arises from the base of the tongue, features cervical lymph node metastases at presentation, and exhibits optically clear nuclei, reminiscent of papillary thyroid cancer [20]. Moreover, in CAMSG, myoepithelial cells are focally present $[18,19]$. We included only PLGA and excluded lesions with features of CAMSG or with lymph node metastasis at diagnosis.

Our findings shed some light on the possible route through which PLGA lesions grow. As the name suggests, this tumor is of low grade of malignancy, because very few cases with metastatic or recurrent disease have been reported. PLGA may, however, grow to cause considerable local destruction, especially as it affects the palate more than any other intraoral site. Mutilating treatment may contribute to local destruction.

Neo-angiogenesis has been extensively investigated in many tumor types, aiming to identify those that might benefit from antiangiogenic treatment [21]. Our PLGA cases were well vascularized, containing numerous $\mathrm{CD} 34$-positive blood vessels surrounding the tumor epithelial cell masses. CD105 immunostaining, however, revealed that most of these vessels were preexisting rather than neo-angiogenic, suggesting that PLGA does not induce neo-angiogenesis. PLGA may instead capitalize on other sources of nutrition to maintain a steady growth.

Metastatic dissemination through the lymphatic system represents one of the major routes through which neoplastic 
disease reaches distant organs, and it is also a key element in determining prognosis [6]. Our results show that lymphatic vessels are scarce and whenever present, confined to the periphery of the lesions. The combination of low neoangiogenesis and poor lymphatic vascularization may explain why metastasis is a rare finding in PLGA.

We found Bcl2 to be strongly and uniformly expressed throughout the PLGA biopsies, whereas Bax expression was weaker and heterogeneous, often within the same lesion. Bax is a pro-apoptotic protein, which is inhibited by $\mathrm{Bcl} 2$ in order to bypass cell death. This suggests that rather than the expressed quantity of either protein, the balance between them will determine whether a cell will live or die [22]. As a consequence, the ratio between the level of expression of $\mathrm{Bax}$ and $\mathrm{Bcl} 2$ might be more informative of the biological behavior of the tumor than that of each marker alone. This is corroborated by the findings from our study, which strongly suggest a shift towards antiapoptosis. In support of this notion, a recent study showed that $\mathrm{Bcl} 2$ does not only prevent apoptosis but, in some tumors, also promotes cell migration and invasion [23].

We also studied cell survival through expression of survivin, which appeared to be strongly expressed in both the nucleus and cytoplasm of PLGA cells. In the cytoplasm, survivin acts as an inhibitor of apoptosis, whereas in the nucleus it is an effector of mitosis and promotes tumor cell proliferation [24]. Only two studies reporting survivin expression in salivary gland tumors have been published, one by Ko et al. [25] focusing on adenoid cystic carcinoma and the other by Ettl et al. [26] on survivin expression in 286 salivary gland carcinomas, including 11 cases of PLGA. Both concluded that nuclear expression of survivin is associated with high-grade malignancy. Since the latter study identified survivin expression in the nucleus, it is conceivable that the location of expression of a protein depends upon the tumor type on which the study was performed. This implies that data on immunohistochemical expression should be cautiously interpreted, and should include other biological mechanisms that stimulate tumor growth. For PLGA, our findings strongly suggest that apoptosis is properly regulated while cell proliferation might be stimulated by Bcl2 and nuclear expression of survivin.

We also investigated cell death via autophagy, using Beclin and LC3B as biomarkers. Autophagy is an important mechanism of cell survival under nutrient and energy starvation and hypoxia $[7,27]$. Our results show that both autophagy markers are strongly expressed in PLGA cells, suggesting that in PLGA autophagy is an important mechanism to support tumor growth. Therefore, therapeutic strategies designed to control or inhibit autophagy ought to be considered, as has been proposed for other tumors [13].

Several studies have focused on cross-talk between apoptosis and autophagy, as comprehensively discussed in the review by El-Khattouti et al. [28]. Suppression of apoptosis induces autophagy and inhibition of autophagy leads to apoptosis, key aspects of tumor behavior to be taken into account when planning treatment.

Senescence has been suggested as the resultant of tumor suppressor pathways. Physiologically, in aging cells, shortening telomeres activate the p53-p21-pRB pathway, whereas in neoplasia this pathway is activated through p16-pRB. Conceivably, p21 may transiently inhibit pRB, with p16 inducing a state of permanent pRB hypophosphorylation [29, 30]. This provided the rationale for our investigation on expression of p21 and p16 in PLGA, even though they are not regarded as specific markers of senescence. Our results show that PLGA cells do not express p 21 and only a small proportion of lesions express p16, though weak at best $(<50 \%)$. These findings suggest that abrogation of senescence pathways may be an additional route to PLGA growth.

We investigated vascularization of PLGA and found it to be well vascularized but not dependent on newly formed vessels. PLGA appear to grow at the expense of autophagy, sustained by anti-apoptotic and anti-senescence signals and stimulated by $\mathrm{Bcl} 2$ and survivin, the former providing a link to cell migration and invasion and the latter to proliferation.

In summary, the findings from this study provide new insight into the natural history of PLGA, which ought to be taken into consideration when designing novel therapeutic strategies. These should reduce mutilating approaches, which will improve the quality of life of patients diagnosed with this disease.

Acknowledgements The authors would like to thank Jeruza Bossonaro and Nadir Freitas for their technical expertise.

Authors' contributions All authors contributed equally to the preparation of the article.

Compliance with ethical standards This study was approved by the Ethics Committee of the São Leopoldo Mandic Institute and Research Center (number 916.794).

Funding The authors are also extremely grateful to FAPESP (São Paulo Research Foundation) and $\mathrm{CNPq}$ for their financial support (FAPESP \#2015/12418-5 and CNPQ \#304031/2014-3).

Conflict of interest The authors declare that they have no conflict of interest.

\section{References}

1. Luna MA, Wenig BM (2005) Polymorphous low-grade adenocarcinoma. In: Barnes L, Eveson JW, Reichart P, Sidransky D, Ed. Pathol. Genet. Head Neck Tumours. pp 223-224

2. Freedman PD, Lumerman H (1983) Lobular carcinoma of intraoral minor salivary gland origin. Oral Surgery, Oral Med Oral Pathol 56: 157-165. doi:10.1016/0030-4220(83)90282-7

3. Batsakis JG, Pinkston GR, Luna MA et al (1983) Adenocarcinomas of the oral cavity: a clinicopathologic study of terminal duct carcinomas. J Laryngol Otol 97:825-835. doi:10.1017/ S0022215100095062 
4. El-Naaj IA, Leiser Y, Wolff A, Peled M (2011) Polymorphous low grade adenocarcinoma: case series and review of surgical management. J Oral Maxillofac Surg 69:1967-1972. doi:10.1016/j.joms. 2010.10.010

5. Carmeliet P, Jain RK (2000) Angiogenesis in cancer and other diseases. Nature 407:249-257. doi:10.1038/35025220

6. Detmar M, Hirakawa S (2002) The formation of lymphatic vessels and its importance in the setting of malignancy. J Exp Med 196:713-718

7. Ouyang L, Shi Z, Zhao S et al (2012) Programmed cell death pathways in cancer: a review of apoptosis, autophagy and programmed necrosis. Cell Prolif 45:487-498. doi:10.1111/j.13652184.2012.00845.x

8. Townson JL, Naumov GN, Chambers AF (2003) The role of apoptosis in tumor progression and metastasis. Curr Mol Med 3:631-642

9. Roberg K, Jonsson A-C, Grénman R, Norberg-Spaak L (2007) Radiotherapy response in oral squamous carcinoma cell lines: evaluation of apoptotic proteins as prognostic factors. Head Neck 29: 325-334. doi:10.1002/hed

10. Levine B (2007) Cell biology: autophagy and cancer. Nature 446: 745-747. doi:10.1038/446745a

11. Lindqvist LM, Simon a K, Baehrecke EH (2015) Current questions and possible controversies in autophagy. Cell Death Discov 1: 15036. doi:10.1038/cddiscovery.2015.36

12. Cosway B, Lovat $P(2016)$ The role of autophagy in squamous cell carcinoma of the head and neck. Oral Oncol 54:1-6. doi:10.1016/j. oraloncology.2015.12.007

13. Burada F, Nicoli ER, Ciurea ME et al (2015) Autophagy in colorectal cancer: an important switch from physiology to pathology. World J Gastrointest Oncol 7:271-284. doi:10.4251/wjgo.v7.i11.271

14. Parkhitko AA, Favorova OO, Henske EP (2013) Autophagy: mechanisms, regulation, and its role in tumorigenesis. Biochemistry 78 : 355-367. doi:10.1134/S0006297913040044

15. Campisi J, d'Adda di Fagagna F (2007) Cellular senescence: when bad things happen to good cells. Nat Rev Mol Cell Biol 8:729-740. doi:10.1038/nrm2233

16. Collado M, Serrano M (2010) Senescence in tumours: evidence from mice and humans. Nat Rev Cancer 10:51-57. doi:10.1038/ $\operatorname{nrc} 2772$

17. Kuilman T, Michaloglou C, Mooi WJ, Peeper DS (2010) The essence of senescence. Genes Dev 24:2463-2479. doi:10.1101/gad. 1971610

18. Michal M, Skálová A, Simpson RHW et al (1999) Cribriform adenocarcinoma of the tongue: a hitherto unrecognized type of adenocarcinoma characteristically occurring in the tongue. Histopathology 35:495-501. doi:10.1046/j.1365-2559.1999. 00792.x

19. Skalova A, Sima R, Kaspirkova-Nemcova J et al (2011) Cribriform adenocarcinoma of minor salivary gland origin principally affecting the tongue: characterization of new entity. Am J Surg Pathol 35: 1168-1176. doi:10.1097/PAS.0b013e31821e1f54

20. Weinreb I, Zhang L, Tirunagari LMS et al (2014) Novel PRKD gene rearrangements and variant fusions in cribriform adenocarcinoma of salivary gland origin. Genes Chromosom Cancer 53:845856. doi:10.1002/gcc. 22195

21. Carmeliet P, Jain RK (2011) Molecular mechanisms and clinical applications of angiogenesis. Nature 473:298-307. doi:10.1038/ nature 10144

22. Wong RSY (2011) Apoptosis in cancer: from pathogenesis to treatment. J Exp Clin Cancer Res 30:87. doi:10.1186/1756-9966-30-87

23. Um H-D (2016) Bcl-2 family proteins as regulators of cancer cell invasion and metastasis: a review focusing on mitochondrial respiration and reactive oxygen species. Oncotarget 7:5193-5203. doi: 10.18632/oncotarget.6405

24. Hirano H, Yamaguchi T, Yokota S et al (2015) Survivin expression in lung cancer: association with smoking, histological types and pathological stages. Oncol Lett 10:1456-1462

25. Ko YH, Roh SY, Won HS et al (2010) Survivin expression in resected adenoid cystic carcinoma of the head and neck. Head Neck Oncol 30:30

26. Ettl T, Stiegler C, Zeitler K et al (2012) EGFR, HER2, survivin, and loss of pSTAT3 characterize high-grade malignancy in salivary gland cancer with impact on prognosis. Hum Pathol 43:921-931. doi:10.1016/j.humpath.2011.08.006

27. Kondo Y, Kanzawa T, Sawaya R, Kondo S (2005) The role of autophagy in cancer development and response to therapy. Nat Rev Cancer 5:726-734. doi:10.1038/nrc1692

28. El-Khattouti A, Selimovic D, Haikel Y, Hassan M (2013) Crosstalk between apoptosis and autophagy: molecular mechanisms and therapeutic strategies in cancer. J Cell Death 6:37-55. doi:10.4137/ JCD.S11034

29. Beauséjour CM, Krtolica A, Galimi F et al (2003) Reversal of human cellular senescence: roles of the p53 and p16 pathways. EMBO J 22:4212-4222. doi:10.1093/emboj/cdg417

30. Dimri GP (2005) What has senescence got to do with cancer? Cancer Cell 7:505-512. doi:10.1016/j.ccr.2005.05.025 